\section{Comparación de la forma de arco anteroinferior individualizado con el método de selección mandibular de Interlandi en modelos con oclusión normal}

\author{
Comparisonoftheanteroiferiorarch'sshapewithmandibularselection \\ individualized method of Interlandi on models with normal occlusion
}

\begin{abstract}
Resumen
El objetivo del estudio es encontrar la forma de arco anteroinferior en oclusiones naturales normales y relacionar este hallazgo con el método de Interlandi de selección del arco anterior mandibular. La muestra fue 9 modelos de estudio de la arcada inferior ( 5 varones y 4 mujeres entre 13-17 años) en normooclusión, con apiñamiento leve, sin faceta de desgaste, sin fracturas coronarias, sin caries ni restauraciones y sin algún tipo de anomalía dentaria en forma, tamańo y número. Se realizó la digitalización de los modelos (con puntos de referencia en cúspide de caninos, punto interincisivo y cara distal de caninos) con un papel milimetrado (para el calibrado en el software Corel Draw x5), con mismas condiciones (brillo, contraste, ampliación al 100\%) y guardados en formato jpg. Una vez calibrada la imagen del modelo, se alineó las cúspides de los caninos en el plano horizontal. Se realizó la primera técnica con la formación del segmento de arco individualizado para cada modelo, formado a partir de 3 puntos no alineados (2 puntos: cúspides de caninos y 1 punto: punto interincisivo); Se midió el radio, ángulo, distancia intercanina, y altura del segmento de arco. Así mismo la segunda técnica con método de Interlandi; eligiendo la curva que mejor se adapte de canino a canino, y en cada curva fue trazada las líneas: intercanina, altura del segmento de arco, midiéndose el radio, ángulo, distancia intercanina, y altura del segmento de arco. En esta técnica, se respetó la longitud de arco de la cartilla de Interlandi, y se trazó desde la cara distal de los caninos hacia el origen del segmento de arco de Interlandi, formando el ángulo intercanino total en la plantilla de interlandi y se midió el ángulo total intercanino. Los datos fueron procesados con el SPSS 15, evaluando para cada variable (media, desviación estándar, mínimo, máximo), entre variables en la primera técnica un análisis bivariada (Correlación de Pearson) y comparando las dos técnicas en muestras relacionadas ( $\mathrm{t}$ de Student). Los resultados obtenidos para el radio del segmento de Arco anterior tiene un promedio de $20,73 \mathrm{~mm} \pm 3,76$, comparado al radio del segmento en Interlandi con $20,66 \mathrm{~mm}$ $\pm 3,67$, el ángulo del segmento de Arco anterior tiene un promedio de $87,48^{\circ} \pm 11,54$, comparado al ángulo del segmento en Interlandi con $87,68^{\circ} \pm 11,27$, la distancia intercanina en el segmento de Arco anterior tiene un promedio de $28,05 \mathrm{~mm} \pm 1,75$, comparado a la distancia intercanina del segmento en Interlandi con $28,04 \mathrm{~mm} \pm 1,78$, la altura del segmento de Arco anterior tiene un promedio de $5,61 \mathrm{~mm}$ $\pm 0,64$, comparado a la altura del segmento en Interlandi con 5,62 $\mathrm{mm} \pm 0,62$. La correlación entre las variables dentro del segmento de arco anterior, se encontró que existe una fuerte correlación entre el radio y el ángulo, el radio y la distancia intercanina, el radio y la altura, así mismo el ángulo y la distancia intercanina, el ángulo y la altura, pero no existe una correlación entre la distancia intercanina y la altura. Al comparar ambos métodos se observa que no existe una diferencia estadísticamente significativa para el radio, el ángulo, la distancia intercanina y la altura del segmento de arco anterior con los arcos anteriores de Interlandi, que no habría diferencia alguna en usar los diagramas de interlandi para la selección del arco anterio-inferior. Ya que existe diferencia en el ángulo del arco antero-inferior, se observó las diferencia que existe entre el ángulo del segmento de distal de caninos dentro de Interlandi con un promedio de $110,11^{\circ} \pm 14,05$ y el ángulo de Interlandi que propone en su diagrama de $120^{\circ}$, siendo esto estadísticamente significante entre ambas medidas.
\end{abstract}

Palabras clave: Arco dental, modelos dentales, ortodoncia, maloclusión

\section{Abstract}

The aim of the study is to find the shape of arch anteroinferior in normal natural occlusions and relate this finding with the Interlandi's method of selection of the mandibular anterior arch. The sample was 9 study models of the lower arch (5 males and 4 females between 13-17 years) in normooclusion, with mild crowding, no facet of wear, without coronary fracture, no decay or restorations and without some kind of tooth abnormality in shape, size and number. It was the digitizing of models (with points of
Artículo Original

\section{Gregorio Javier Carhuamaca León ${ }^{1}$, Ana María Díaz Soriano ${ }^{2}$}

1Residente de la Segunda Especialidad de Ortodoncia de la Facultad de Odontología de la UNMSM.

2Docente Segunda Especialidad de OrtodonciaAsignatura Tesis Facultad Odontología UNMSM.

Correspondencia 1:

Asociación "Villa Rica” Mz "O” Lote 34 Chaclacayo Lima-Perú

Celular: 997497868

e-mail: odonto_javier@hotmail.com/ortodoncia. new.smile@gmail.com 
reference on cusp of canines, interincisor point and canine distal face) with a graph paper (for the calibration software Corel Draw x 5), with same conditions (brightness, contrast, extension 100) and saved in jpg format. Once calibrated the model image, aligned the cusps of the canines in the horizontal plane. First technique with the formation of the segment arch individualized for each model, formed from three unaligned points ( 2 points: cusps of canines and 1 point: interincisivo point); Measured the radius, angle, intercanin distance, and height of the arc segment. In this technique, Interlandi primer arc length, was respected and was drawn from the distal face of canines to the origin of the Interlandi arc segment, the total intercanin angle on the template of interlandi and measured total intercanin angle. The data were processed with SPSS 15, evaluating a bivariate analysis (Pearson correlation) for each variable (average, standard deviation, minimum, maximum), between variables in the first technique and comparing the two techniques in related samples (Student's t). The results obtained for the radius of the previous arc segment has an average of $20,73 \mathrm{~mm} \pm 3.76$, compared to the radius of the segment in Interlandi with $20,66 \mathrm{~mm} \pm 3.67$, the angle of the anterior arch segment averages $87,48^{\circ} \pm 11.54$, compared to the angle of the segment in Interlandi with $87,68^{\circ} \pm 11.27$, distance intercanin in the anterior arch segment has an average of $28,05 \mathrm{~mm} \pm 1.75$ compared to the intercanin away from the segment in Interlandi 28 , $04 \mathrm{~mm} \pm 1.78$, the height of the anterior arch segment has an average of $5,61 \mathrm{~mm} \pm 0.64$, compared to the height of the segment in Interlandi $5,62 \mathrm{~mm} \pm 0.62$. The correlation between the variables within the anterior arch segment, found that there is a strong correlation between radius and angle, radius and intercanin distance, radius and height, the same angle and intercanin distance, angle and height, but there is a correlation between the intercanin distance and height. Comparing both methods shows that there is a statistically significant difference for the radius, the angle, the intercanin distance and height of segment of anterior arch with the previous arcs of Interlandi, that there would be no difference any use interlandi diagrams for the selection of the anterio-lower arch. Counter there is difference in the angle of the arc, was the difference that exists between the angle of the segment distal to canines within Interlandi with an average of $110,11^{\circ} \pm 14,05$ mean, and Interlandi's angle which proposes in its diagram of 120 ${ }^{\circ}$, this being statistically significant between the two measures.

Keywords: Dental arch, dental models, orthodontics, malocclusion.

\section{Introducción}

El estudio de la arcada dental, de su forma y su métrica, es muy importante en odontología general, ortodoncia, prótesis o implantología, odontología forense, antropología y paleoantropología. La capacidad para describir la forma de la arcada dental es necesaria para los estudios biomecánicos de oclusión, así como para los estudios antropológicos y de variación dental en humanos y primates o para los estudios clínicos previos y post tratamiento ortodóntico.

La morfología del arco dental es una consideración importante en el tratamiento de ortodoncia de las deformidades dentofaciales. Desde hace más de un siglo la morfología del arco dental ha sido estudiada con la esperanza de definir los objetivos adecuados para la posición de los dientes, la estética, función y estabilidad a largo plazo. El arco dental mandibular, es una de las principales referencias para la planificación del tratamiento ortodóncico, por ello muchos estudios se han esforzado por definir su tamaño ideal y su morfología.

De acuerdo con la literatura, la morfología del arco dental humano muestra una variación individual de ancho. Las descripciones varían desde los trabajos de Hawley1 y su primer intento por describir la forma de la arcada dental, asimismo, muchos autores han pretendido describir la forma de la arcada y los métodos para su estudio como una forma elipsoidal, parabólico, un segmento de círculo unido a las líneas, un segmento de círculo de curva catenaria, etc.
Muchos diagramas fueron diseñados para ayudar a los ortodoncistas en la formación de arcos durante el tratamiento. El uso de diagramas estandarizados ha sido cuestionado por algunos autores, que sugieren utilizar fórmulas matemáticas para encontrar una forma más individualizada de los arcos.

El objetivo de este estudio fue encontrar la forma de arco anteroinferior (de canino a canino) en oclusiones naturales normales y luego relacionar este hallazgo con el método de Interlandi para la selección del arco anterior mandibular para la selección de plantillas propuestos por el autor.

\section{Material y métodos}

\section{Muestra}

La muestra consta de 9 modelos de estudio del arco dental inferior, de 5 varones y 4 mujeres cuyas edades oscilan entre 13-17 años con normooclusión, apińamiento dentario leve, sin faceta de desgaste (oclusal e interproximal), sin fracturas coronarias, sin caries, ni restauraciones (oclusal e interproximal) y sin algún tipo de anomalía dentaria (forma, tamaño y número).

\section{Recolección de datos}

Los sistemas informáticos utilizados para el registro, procesado y evaluación de nuestra muestra fueron: Sistema Operativo Windows 7, Microsoft Word (Office 2010), Corel Draw x5, Hp Scaner 4400c, SPSS 15.
Fecha de recepción: 08 de abril

Fecha de aceptación: 14 de junio
Se marcaron los puntos de referencia, se siguió con el mismo sistema, en todos los modelos de estudio, estas marcas utilizadas se basaron en estudios previos, estos fueron: cúspide de caninos, punto interincisivo y cara distal de caninos. (Fig. 1).

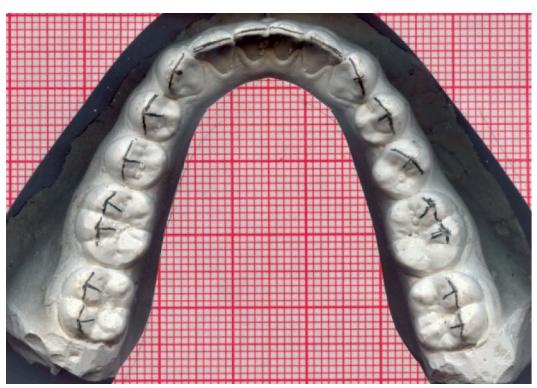

Fig. 1. Modelo inferior con puntos de referencia, escaneado y calibrado milimétricamente.

Se realizó digitalizaciones de los modelos con un papel milimetrado (para el calibrado), siempre en las mismas condiciones (brillo, contraste, ampliación al $100 \%$ ) en formato jpg. Hubo calibrado de las digitalizaciones en el software Corel Draw x5, utilizando la imagen milimetrada en una proporción de $1: 1$. (fig. 1). Una vez calibrada la imagen del modelo de estudio, se alineó las cúspides de los caninos en el plano horizontal.

Se utilizó la primera técnica con la formación del segmento de arco individualizado para cada modelo, formado a partir de 3 puntos no alineados (2 
puntos A y C: cúspides de caninos y 1 punto $\mathrm{B}$ : punto interincisivo); primero se unen los 3 puntos, dos a dos, (A-B y B-C), luego se trazan las mediatrices de los segmentos $\mathrm{AB}$ y $\mathrm{BC}$, posteriormente el punto $\mathrm{O}$, donde se cortan las 2 mediatrices, es el centro del arco solicitado. Desde este punto se traza el arco circular que deberá pasar por los 3 puntos. (Figs. 2 y 3 ).

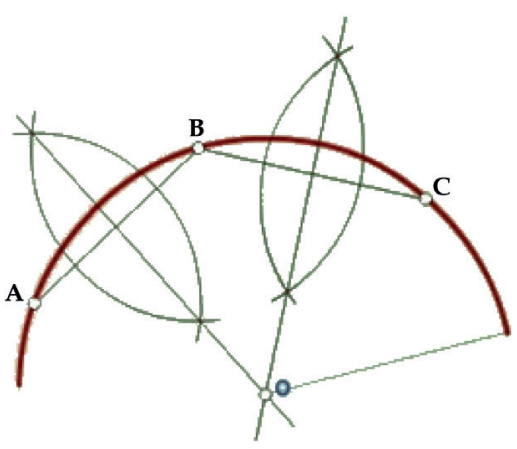

Fig 2. Confección de un segmento de arco circular a partir de tres puntos no congruentes.

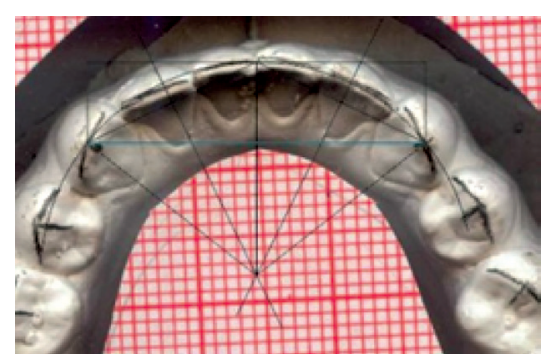

Fig 3. Confección de un segmento de arco circular a partir de tres puntos no congruentes en el modelo inferior escaneado y calibrado realizado en Corel Draw $x 5$.

Se midió el radio, ángulo, distancia intercanina, y altura del segmento de arco. Posteriormente la segunda técnica se realizó según el método de elección de arco anterior de Interlandi, reproduciendo en el software los gráficos del arco anterior con un radio de 16 a 26 $\mathrm{mm}$ y con un ángulo de $120^{\circ}$ (descrito así por Bonwill). Se eligió la curva que mejor se adaptaba de canino a canino, y en cada curva se trazó las líneas: intercanina, altura del segmento de arco. Se midió el radio, ángulo, distancia intercanina, y altura del segmento de arco. En esta técnica, respetando la longitud de arco a $120^{\circ}$ propio de la cartilla de Interlandi, se traza desde la cara distal de los caninos hacia el origen del segmento de arco de Interlandi, formando el ángulo intercanino total en la plantilla de interlandi. Se midió el ángulo total intercanino.

\section{Resultados}

Determinación del segmento de arco anterior individualizado.

El comportamiento de las variables en cada método, no tiene mucha variación en cuanto a sus valores. (Tabla 1 )

Tabla 1. Promedio, Desviación Estándar del radio, ángulo distancia intercanina y altura del Segmento de Arco Anterior diseńado por tres puntos y en el segmento de arco anterior de Interlandi.

\begin{tabular}{lccccc}
\hline & N & Mín & Máx & Med & Desv. típ. \\
\hline Radio del Segmento de Arco Anterior & 9 & 16,03 & 29,43 & 20,73 & 3,76 \\
Angulo del Segmento de Arco Anterior & 9 & 62,41 & 103,18 & 87,48 & 11,54 \\
$\begin{array}{l}\text { Distancia Intercanina en Segmento de Arco } \\
\text { Anterior }\end{array}$ & 9 & 25,11 & 30,49 & 28,05 & 1,75 \\
$\begin{array}{l}\text { Altura del Segmento de Arco Anterior } \\
\text { Radio del Segmento en Interlandi }\end{array}$ & 9 & 4,26 & 6,26 & 5,61 & 0,64 \\
Angulo del Segmento en Interlandi & 9 & 16,00 & 29,00 & 20,66 & 3,67 \\
Distancia Intercanina en Interlandi & 9 & 25,10 & 30,43 & 28,04 & 1,78 \\
Altura del Segmento en Interlandi & 9 & 4,31 & 6,31 & 5,62 & 0,62 \\
\hline
\end{tabular}

El radio del segmento de Arco anterior tiene un promedio de 20,73 $\mathrm{mm} \pm 3,76$, comparado al radio del segmento en Interlandi con $20,66 \mathrm{~mm} \pm 3,67$.

El ángulo del segmento de Arco anterior tiene un promedio de 87,48mm $\pm 11,54$, comparado al ángulo del segmento en Interlandi con $87,68 \mathrm{~mm} \pm 11,27$.

La distancia intercanina en el segmento de Arco tiene un promedio de $28,05 \mathrm{~mm}$ $\pm 1,75$, comparado a la distancia intercanina del segmento en Interlandi con $28,04 \mathrm{~mm} \pm 1,78$.

La altura del segmento de Arco anterior tiene un promedio de 5,61 $\mathrm{mm} \pm 0,64$, comparado a la altura del segmento en Interlandi con 5,62 $\mathrm{mm} \pm 0,62$.

Relación entre las variables en el Segmento de Arco Anterior

Las variables dentro del Segmento de Arco Anterior, conformado por el radio, ángulo, distancia intercanina y la altura, tienen una relación entre ellas por la cual se buscó la correlación existente entre estas variable: (Tablas 2,3,4,5,6,7)

En la tabla 2 existe una fuerte correlación entre el Radio y el ángulo del Segmento de Arco Anterior, esto se debe a que si el ángulo disminuye, el radio aumenta; así mismo ocurre si el ángulo aumenta el radio disminuirá; pero estos dos dependerán del comportamiento de la distancia intercanina y de la altura del segmento de arco anterior.

Tabla 2. Correlación entre el radio y el ángulo del Segmento de Arco Anterior diseñado por tres puntos.

\begin{tabular}{lccc}
\hline & & $\begin{array}{c}\text { Radio del Segmento } \\
\text { de Arco Anterior }\end{array}$ & $\begin{array}{c}\text { Angulo del Segmento } \\
\text { de Arco Anterior }\end{array}$ \\
\hline $\begin{array}{l}\text { Radio del Segmento de } \\
\text { Arco Anterior }\end{array}$ & $\begin{array}{c}\text { Correlación de } \\
\text { Pearson } \\
\text { Sig. (bilateral) }\end{array}$ & 1 & $\left.-0,9611^{* *}\right)$ \\
$\begin{array}{l}\text { Angulo del Segmento } \\
\text { de Arco Anterior }\end{array}$ & $\begin{array}{c}\text { Correlación de } \\
\text { Pearson }\end{array}$ & $\left.-0,9611^{* *}\right)$ & 0,000 \\
& Sig. (bilateral) & 0,000 & 1 \\
\hline
\end{tabular}

** La correlación es significativa al nivel 0,01 (bilateral). 
En la tabla 3 existe una fuerte correlación entre el radio y la distancia intercanina del Segmento de Arco Anterior, esto se debe a que si el radio disminuye, la distancia intercanina disminuirá; así mismo si el radio aumenta, la distancia intercanina aumentará, esto se convierte en una condicional de que el arco anterior sea pequeño o grande.

En la tabla 4 existe una fuerte correlación entre el Radio y la altura del Segmento de Arco Anterior; esto se debe a que si la altura disminuye, el radio aumenta; así mismo ocurre si la altura aumenta, el radio disminuye, por la que esto se convierte en una condicional de que el arco anterior sea más ovalado o estrecho correspondientemente.

En la tabla 5, se observa que existe una fuerte correlación entre el ángulo y la distancia intercanina del Segmento de Arco Anterior, esto se debe a que si la distancia intercanina aumenta, así mismo el ángulo del segmento también aumentaría, por tanto el segmento de arco anterior aumentará.

En la tabla 6 existe una fuerte correlación entre el ángulo y la altura del Segmento de Arco Anterior, esto se debe a que si el ángulo disminuye, la altura aumenta; así mismo si el ángulo aumenta, la altura disminuye, esto condiciona a que el arco anterior sea estrecho o amplio respectivamente.

En la tabla 7, se observa que no existe una correlación entre la distancia intercanina y la altura del Segmento de Arco Anterior; esto se debería a que la distancia intercanina con la altura están relacionados íntimamente.

Para determinar el ángulo, el radio, y la longitud de arco, mientras estos valores varían nos darán tanto segmentos de arcos amplios o estrechos.

Comparación de los dos métodos de forma de arco anterior

En la tabla 8 describe las diferencias que existen entre cada variable de ambos métodos de obtención de forma de arco antero-inferior.

Se observa que no existe una diferencia estadísticamente significativa para el radio, el ángulo, la distancia intercanina y la altura del segmento de arco anterior con los arcos anteriores de Interlandi.

Esto nos hace suponer que no habría diferencia alguna en usar los diagramas de interlandi para la selección del arco antero-inferior, tanto estadísticamente como clínicamente no hay una diferencia relevante, cabe resaltar que esto se cumple en la muestra estudiada, que son modelos de estudio en normo-oclu-
Tabla 3. Correlación entre el radio y la distancia intercanina del Segmento de Arco Anterior diseńado por tres puntos.

\begin{tabular}{lccc}
\hline & & $\begin{array}{c}\text { Radio del Segmento } \\
\text { de Arco Anterior }\end{array}$ & $\begin{array}{c}\text { Distancia Intercanina } \\
\text { en Segmento de Arco } \\
\text { Anterior }\end{array}$ \\
\hline $\begin{array}{l}\text { Radio del Segmento } \\
\text { de Arco Anterior }\end{array}$ & $\begin{array}{c}\text { Correlación de } \\
\text { Pearson } \\
\text { Sig. (bilateral) }\end{array}$ & 1 & $0,814\left(^{* *}\right)$ \\
$\begin{array}{l}\text { Distancia Intercanina } \\
\text { en Segmento de Arco } \\
\text { Anterior }\end{array}$ & $\begin{array}{c}\text { Correlación de } \\
\text { Pearson }\end{array}$ & $0,814\left(^{* *}\right)$ & 0,008 \\
& Sig. (bilateral) & 0,008 & 1 \\
\hline
\end{tabular}

** La correlación es significativa al nivel 0,01 (bilateral).

Tabla 4. Correlación entre el radio y la altura del Segmento de Arco Anterior disenado por tres puntos.

\begin{tabular}{lccc}
\hline & & $\begin{array}{c}\text { Radio del Segmento } \\
\text { de Arco Anterior }\end{array}$ & $\begin{array}{c}\text { Altura del Segmento de } \\
\text { Arco Anterior }\end{array}$ \\
\hline $\begin{array}{l}\text { Radio del Segmento de } \\
\text { Arco Anterior }\end{array}$ & $\begin{array}{c}\text { Correlación de } \\
\text { Pearson } \\
\text { Sig. (bilateral) }\end{array}$ & 1 & $-0,791\left(^{*}\right)$ \\
$\begin{array}{l}\text { Altura del Segmento de } \\
\text { Arco Anterior }\end{array}$ & $\begin{array}{c}\text { Correlación de } \\
\text { Pearson }\end{array}$ & $-0,791\left(^{*}\right)$ & 0,011 \\
& Sig. (bilateral) & 0,011 & 1 \\
\hline
\end{tabular}

* La correlación es significante al nivel 0,05 (bilateral).

Tabla 5. Correlación entre el ángulo y la distancia intercanina del Segmento de Arco Anterior diseńado por tres puntos.

\begin{tabular}{lccc}
\hline & & $\begin{array}{c}\text { Angulo del Segmento } \\
\text { de Arco Anterior }\end{array}$ & $\begin{array}{c}\text { Distancia Intercanina } \\
\text { en Segmento de Arco } \\
\text { Anterior }\end{array}$ \\
\hline $\begin{array}{l}\text { Angulo del Segmento } \\
\text { de Arco Anterior }\end{array}$ & $\begin{array}{c}\text { Correlación de } \\
\text { Pearson } \\
\text { Sig. (bilateral) }\end{array}$ & 1 & $-0,682\left(^{*}\right)$ \\
$\begin{array}{l}\text { Distancia Intercanina } \\
\text { en Segmento de Arco } \\
\text { Anterior }\end{array}$ & $\begin{array}{c}\text { Correlación de } \\
\text { Pearson }\end{array}$ & $-0,682\left(^{*}\right)$ & 0,043 \\
& Sig. (bilateral) & 0,043 & 1 \\
\hline
\end{tabular}

* La correlación es significante al nivel 0,05 (bilateral).

Tabla 6. Correlación entre el ángulo y la altura del Segmento de Arco Anterior diseñado por tres puntos.

\begin{tabular}{lccc}
\hline & & $\begin{array}{c}\text { Angulo del Segmento } \\
\text { de Arco Anterior }\end{array}$ & $\begin{array}{c}\text { Altura del Segmento } \\
\text { de Arco Anterior }\end{array}$ \\
\hline $\begin{array}{l}\text { Angulo del Segmento } \\
\text { de Arco Anterior }\end{array}$ & $\begin{array}{c}\text { Correlación de } \\
\text { Pearson } \\
\text { Sig. (bilateral) }\end{array}$ & 1 & $0,908\left(^{* *}\right)$ \\
$\begin{array}{l}\text { Altura del Segmento de } \\
\text { Arco Anterior }\end{array}$ & $\begin{array}{c}\text { Correlación de } \\
\text { Pearson }\end{array}$ & $0,908\left({ }^{* *}\right)$ & 0,001 \\
& Sig. (bilateral) & 0,001 & 1 \\
\hline
\end{tabular}

** La correlación es significativa al nivel 0,01 (bilateral). 
Tabla 7 Correlación entre la distancia intercanina y la altura del Segmento de Arco Anterior diseñado por tres puntos.

\begin{tabular}{lccc}
\hline & \multicolumn{1}{c}{$\begin{array}{c}\text { Distancia Intercanin } \\
\text { Segmento de Arco Anterior }\end{array}$} & $\begin{array}{c}\text { Altura del Segmento } \\
\text { de Arco Anterior }\end{array}$ \\
\hline $\begin{array}{l}\text { Distancia Intercanin } \\
\begin{array}{l}\text { Segmento de Arco } \\
\text { Anterior }\end{array}\end{array}$ & $\begin{array}{c}\text { Correl de } \\
\text { Pearson }\end{array}$ & 1 & $-0,315$ \\
& Sig. (bilateral) & & 0,409 \\
$\begin{array}{l}\text { Altura del Segmento } \\
\text { de Arco Anterior }\end{array}$ & $\begin{array}{c}\text { Correlación de } \\
\text { Pearson } \\
\text { Sig. (bilateral) }\end{array}$ & $-0,315$ & 1 \\
& 0,409 & \\
\hline
\end{tabular}

Tabla 8. Comparación de los métodos de segmento de arco anterior diseñado por tres puntos y el segmento de arco anterior de Interlandi.

\begin{tabular}{|c|c|c|c|c|c|c|c|c|c|}
\hline & & \multicolumn{5}{|c|}{ Diferencias relacionadas } & \multirow{3}{*}{$t$} & \multirow{3}{*}{$\mathrm{gl}$} & \multirow{3}{*}{$\begin{array}{l}\text { Sig. } \\
\text { (bilate- } \\
\text { ral) }\end{array}$} \\
\hline & & \multirow[t]{2}{*}{ Media } & \multirow{2}{*}{$\begin{array}{l}\text { Des- } \\
\text { viación } \\
\text { típ. }\end{array}$} & \multirow{2}{*}{$\begin{array}{l}\text { Error } \\
\text { típ. } \\
\text { de la } \\
\text { media }\end{array}$} & \multicolumn{2}{|c|}{$\begin{array}{c}95 \% \text { Intervalo de } \\
\text { confianza para la } \\
\text { diferencia }\end{array}$} & & & \\
\hline & & & & & Sup & $\operatorname{lnf}$ & & & \\
\hline Par 1 & $\begin{array}{l}\text { Radio del } \\
\text { Segmento de Arco } \\
\text { Anterior - Radio } \\
\text { del Segmento en } \\
\text { Interlandi }\end{array}$ & 0,0711 & 0,33202 & 0,11067 & $-0,18410$ & 0,3263 & 0,64 & 8 & 0,538 \\
\hline Par 2 & $\begin{array}{l}\text { Angulo del } \\
\text { Segmento de Arco } \\
\text { Anterior - Angulo } \\
\text { del Segmento en } \\
\text { Interlandi }\end{array}$ & $-0,2033$ & 1,26979 & 0,42326 & $-1,17938$ & 0,7727 & $-0,48$ & 8 & 0,644 \\
\hline Par 3 & $\begin{array}{l}\text { Distancia Interca- } \\
\text { nina en Segmento } \\
\text { de Arco Anterior } \\
\text { - Distancia } \\
\text { Intercanina en } \\
\text { Interlandi }\end{array}$ & 0,0133 & 0,12580 & 0,04193 & $-0,08336$ & 0,1100 & 0,31 & 8 & 0,759 \\
\hline Par 4 & $\begin{array}{l}\text { Altura del } \\
\text { Segmento de Arco } \\
\text { Anterior - Altura } \\
\text { del Segmento en } \\
\text { Interlandi }\end{array}$ & $-0,0122$ & 0,06320 & 0,02107 & $-0,06080$ & 0,0363 & $-0,58$ & 8 & 0,578 \\
\hline
\end{tabular}

Tabla 9. Comparación de la longitud de Arco del segmento de Arco anterior diseńado por tres puntos con la longitud de Arco de Interlandi.

\begin{tabular}{lccccc}
\hline & N & Mínimo & Máximo & Media & Desv. típ. \\
\hline Angulo de Interlandi & 9 & 120,00 & 120,00 & 120,00 & 0,00 \\
$\begin{array}{l}\text { Angulo del Segmento de Distal de } \\
\begin{array}{l}\text { Caninos en Interlandi } \\
\hline\end{array}\end{array}$ & 9 & 78,60 & 127,72 & 110,11 & 14,05 \\
\hline
\end{tabular}

Tabla 10. Comparación de la longitud de Arco del segmento de Arco anterior diseńado por tres puntos a nivel de la cara distal de caninos, con la longitud de Arco de Interlandi.

\begin{tabular}{|c|c|c|c|c|c|c|c|c|c|}
\hline & & \multicolumn{5}{|c|}{ Diferencias relacionadas } & \multirow{3}{*}{$\mathrm{t}$} & \multirow{3}{*}{$\mathrm{gl}$} & \multirow{3}{*}{$\begin{array}{l}\text { Sig. } \\
\text { (bila- } \\
\text { teral) }\end{array}$} \\
\hline & & \multirow[t]{2}{*}{ Media } & \multirow[t]{2}{*}{$\begin{array}{l}\text { Desvia- } \\
\text { ción típ. }\end{array}$} & \multirow{2}{*}{$\begin{array}{l}\text { Error } \\
\text { típ. de la } \\
\text { media }\end{array}$} & \multicolumn{2}{|c|}{$\begin{array}{c}95 \% \text { Intervalo de } \\
\text { confianza para la } \\
\text { diferencia }\end{array}$} & & & \\
\hline & & & & & Superior & Inferior & & & \\
\hline Par 1 & $\begin{array}{l}\text { Angulo de Inter- } \\
\text { landi - Angulo } \\
\text { del Segmento de } \\
\text { Distal de Caninos } \\
\text { en Interlandi }\end{array}$ & 9,88667 & 14,05821 & 4,68607 & $-0,919$ & 20,692 & 2,110 & 8 & 0,068 \\
\hline
\end{tabular}

sión y que los puntos seleccionados son las cúspides de los caninos y un punto inter-incisivo.

Esta relación se cumple teniendo en cuenta que el ángulo del arco anterior fluctúa con un promedio de $87,4^{\circ} \pm$ 11,54 y $87,68^{\circ} \pm 11,27$ en ambos métodos (Ver Tabla 1); pero los diagramas de Interlandi basándose en los trabajos de Hawley y de Bonwill, el ángulo del arco antero-inferior es de $120^{\circ}$.

\section{Ajuste del arco de Interlandi}

Debido a la diferencia que existe en el ángulo del arco antero-inferior, se realizó las medidas tomando como base el diagrama de interlandi, y como referencia las caras distales de los caninos, ya que entre esos puntos se determina el sector anterior de la arcada.

En la tabla 9, se observa las diferencia que existe entre el ángulo del segmento de distal de caninos dentro de Interlandi con un promedio de $110,11^{\circ} \pm 14,05$ y el ángulo de Interlandi que propone en su diagrama de $120^{\circ}$.

En la tabla 10 se observa la diferencia estadísticamente significativa entre ambas medidas, corroborando la diferencia clínica

entre ambos, esto nos hace suponer que si existe esta diferencia en el ángulo del arco antero-inferior, existirá una diferencia en la longitud de arco anteroinferior esta a su vez conlleva a concluir que se puede utilizar el diagrama de selección de arco anterior de Interlandi, más no la longitud de arco que presenta el diagrama, y que al usarlo se cometería el error de aumentar la distancia intercanina y la forma de arco que presenta el paciente naturalmente. Llama a la reflexión de modificar el diagrama de Interlandi en cuanto a su ángulo y la longitud de arco antero-inferior. Cabe resaltar que esto se cumple en la muestra estudiada, que son modelos de estudio en normo-oclusión y que los puntos seleccionados son las cúspides de los caninos y un punto inter-incisivo.

\section{Discusión}

Hawley ${ }^{1}$ que se basó en los trabajos de Bonwill (1885), refiere que la forma de arcada ideal tendría forma de triángulo equilátero, cuya base representa la anchura bicondílea y el vértice al punto interincisivo, y que este triángulo equilátero determina que el ángulo del arco anterior es de $120^{\circ}$, mientras que en la muestra estudiada representa un ángulo de $110,11^{\circ} \pm 14,05$. 
Brader $^{2}$ sugirió que la forma de arcada dental es producto de un equilibrio de fuerzas y que se aproxima a la porción más estrecha de una curva de elipse trifocal, estudiando los trabajos de anteriores investigadores, esta elipse trifocal hace que la forma de arco está conformada por tres segmentos de arco: un anterior y dos posteriores. Determinando que el arco anterior es un segmento de arco circular sin especificar el ángulo que forma este segmento, concuerda con este estudio de que el arco anterior es un segmento circular.

Felton y cols. ${ }^{3}$ realizaron un análisis computarizado de la forma y estabilidad mandibular mediante fotocopias de modelos y su digitalización, encontraron que la forma de arco es variable y lo agruparon en 3 grupos (estrecho, normal y ovalado), coincidiendo que el sector anterior tiene un componente de arco circular, coincidente con este estudio en determinar que la forma de arco anterior es un segmento de arco.

Ferrario y cols. ${ }^{4}$ estudiaron mediante el euclidean distance matrix analysis (estudia coordenadas digitalizadas) la forma de arcada dental, concluyendo que la forma de la arcada no es definida por una figura geométrica estandarizada, y que esta pueda variar, así mismo este estudio determina que la forma de arco anterior es variable, en forma y amplitud.

Ferrario y cols. ${ }^{5}$ estudiaron la definición matemática de la forma de arcada en humanos con dentición normal, en adultos jóvenes mediante funciones de cuarto grado y ajuste a elíptica (dientes anteriores) o parabólica (dientes posteriores), diferente a este estudio, en que el arco anterior es segmento circular.

Los hallazgos en este estudio sugieren que es importante mantener la forma de arco individual durante el tratamiento de ortodoncia, ya que este arco es muy variable y dependiente de los biotipos faciales y esqueletales.

En la actualidad existen arcos preformados para el tratamiento de ortodoncia, esto podría estar yendo contra la forma de arco del paciente, ya que se ha observado que existe una variación en el arco antero-inferior, y que este arco es el inicio para determinar la forma de arco total de la arcada, debido a esto es importante realizar la individualización de los arcos y no utilizar arcos preformados.

Con el afán de facilitar y disminuir el tiempo de trabajo del ortodoncista se ha obtenido mediante las empresas de venta de arcos preformados de ortodoncia, pero esto puede ir en contra de la biología y anatomía del paciente.

Si la forma de arco anterior es variable, así mismo puede variar la forma de arco posterior, queda esta como interrogante para próximos estudios.

\section{Conclusiones}

No existe diferencia estadísticamente significativa entre ambos métodos para la selección de forma de arco anterior. Por tanto, es válido usar las plantillas de arco anterior de Interlandi para esta muestra, mas no respetar la longitud de arco.

Existe diferencia estadísticamente significativa para el ángulo y la longitud de arco entre las plantillas de arco anterior de Interlandi y el arco real intercanino (caras distales). Por tanto no es válido respetar la longitud de arco propuesto por interlandi con un ángulo de $120^{\circ}$ en esta muestra.

La discrepancia entre el mínimo, media y máximo valor en el radio, ángulo, distancia intercanina, y altura del segmento de arco en el método geométrico de forma de arco individualizado por 3 puntos no alineados; sugiere una idea de que la variabilidad se debe a una diferencia de biotipos faciales.

\section{Agradecimiento}

A los tutores de la especialidad de Ortodoncia y Ortopedia Maxilar de la Facultad de Odontología de la Universidad Nacional Mayor de San Marcos, por el incentivo y el fomentar la investigación para cubrir las expectativas de diagnóstico, planeamiento y tratamiento en los pacientes con maloclusión, y brindarles una mejor atención y calidad de servicio.

\section{Referencias bibliográficas}

1 Hawley CA. Determination of the normal arch, and its application to orthodontia. Dental Cosmos 1905;47:541-52.

2 Brader AC. Dental arch form related with intraoral forces: $\mathrm{pr}=\mathrm{c}$. Am J Orthod 1972;61:541-60.

3 Felton JM, Sinclair PM, Jones DL, Alexander RG. A computerized analysis of the shape and stability of mandibular arch form. Am J Orthod Dentofac Orthop 1987;92:48-83.

4 Ferrario VF, Sforza C, Miani A, Tartaglia G. Human dental arch shape evaluated by euclidean-distance matrix analysis. Am J Phys Anthropol 1993;90:445-53.

5 Ferrario VF, Sforza C, Miani A, Tartaglia G. Mathematical definition of the shape of dental arches in human permanent healthy dentitions. Eur J Orthod 1994;16:28794.

6 Battagel J. Individualized catenary curves: their relationship to arch form and perimeter. $\mathrm{Br} \mathrm{J}$ Orthod 1996;23:21-8.

7 Steyn CL, Harris AMP, Hons, Du Preez RJ. Anterior arch circumference adjustment - how much? Angle Orthod 1996;66: 457-61.

8 BeGole EA, Lyew RC. A new method for analyzing change in dental arch form. Am J Orthod Dentofac Orthop 1998;113:394401.

9 Visessaksanti U. An easy method of checking archform and canine width. J Clin Orthod 1998;32:60810.

10 Ferrario VF, Sforza C, Poggio CE, Serrao G, Colombo A. Three dimensional dental arch curvature in human adolescents and adults. Am J Orthod Dentofac Orthop 1999;115:401-5. 